\title{
Susy Seesaw Inflation and NMSO(10)GUT
}

\author{
Charanjit S. Aulakh \\ Dept. of Physics, Panjab University, Chandigarh, India, 160014
}

\begin{abstract}
We show that Supersymmetric models with Type I seesaw neutrino masses support slow roll inflection point inflation. The inflaton is the D-flat direction labelled by the chiral invariant HLN composed of the Higgs $(H)$, slepton(L) and conjugate sneutrino(N) superfields. The scale of inflation and fine tuning is set by the conjugate neutrino Majorana mass $M_{V^{c}} \sim 10^{6}-10^{12} \mathrm{GeV}$. The cubic term in the (quartic) inflaton potential is dominantly from superpotential (not soft Susy breaking) couplings. The tuning conditions are thus insensitive to soft supersymmetry breaking parameters and are generically much less stringent than for previous 'A-term' inflation scenarios controlled by mass scales $\sim T e V$. WMAP limits on the ratio of tensor to scalar perturbations limit the scale $M$ controlling inflection point inflation: $M<7.9 \times 10^{13} \mathrm{GeV}$. 'Instant preheating' is operative and dumps the inflaton energy into MSSM modes giving a high reheat temperature : $T_{r h} \approx M_{v^{c}}^{\frac{3}{4}} 10^{6}$ $\mathrm{GeV} \sim 10^{11}-10^{15} \mathrm{GeV}$. A large gravitino mass $>50 \mathrm{TeV}$ is therefore required to avoid over closure by reheat produced gravitinos. 'Instant preheating' and NLH inflaton facilitate production of right handed neutrinos during inflaton decay and thus non-thermal leptogenesis in addition to thermal leptogenesis. We show that the embedding in the fully realistic New Minimal Supersymmetric SO(10) GUT requires use of the heaviest righthanded neutrino mass as the controlling scale but the possibility of a measurable tensor scalar perturbation ratio seems marginal. We examine the parametric difficulties remaining.
\end{abstract}

Keywords: Inflation, Seesaw, Supersymmetry, GUTs, SO(10)

PACS: $12.10 . \mathrm{Dm}, 98.80 . \mathrm{Cq}, 14.60 . \mathrm{Pq}$

\section{INTRODUCTION}

Primordial inflation is now generally accepted as the only viable mechanism for setting the initial conditions for Big bang Cosmogony in a way compatible with the extreme CMB homogeneity observed by increasingly accurate satellite maps [1] of the Microwave sky. The parameters of inflation $\left(P_{R}, n_{s}, \mathscr{D}_{k}\left(n_{s}\right)\right)$ measured so far can be accounted for by most of the many slow-roll inflation models proposed. With few exceptions these models use inflaton(s) that have no role to play outside of inflation since they have no connection with the known fields of Particle Physics. Such a connection is however necessary since the post-inflationary epoch must include reheating phases where the inflaton energy is converted into the matter and radiation observed today. Models driven by an inflaton composed of SM[2],MSSM [3] or GUT[4] fields thus carry an obvious appeal. Models of the second type type are typically based on slow roll inflation along "D-flat directions" in the MSSM field space and these are conveniently labelled by holomorphic gauge invariants formed from chiral superfields. Such models ( also called "A-Term Inflation" models $[5,6,7])$ typically require extreme fine tuning between the soft terms to ensure an inflection or saddle point of the field potential where the vacuum energy density drives a burst of inflation but nevertheless allows "graceful exit" due to the absence of a local minimum and the associated potential barrier which would prevent exit. Thus while they answer some of the relevant issues they have much scope for improvement.

In $[5,6]$ an A-term inflation model was based on small neutrino yukawa couplings needed for realistic Dirac light neutrino masses. The inflaton field was a gauge invariant $D$-flat direction, $N H L$, where $N$ is the right handed sneutrino, $H$ is the MSSM Higgs doublet which gives masses to the up-type quarks, and $L$ is the slepton field. When coupled with soft trilinear and bilinear supersymmetry breaking terms of mass scale $\sim 100 \mathrm{GeV}$ to $10 \mathrm{TeV}$ the associated renormalizable inflaton potential can be fine tuned to achieve inflection point inflation consistent with WMAP 7 year data[5, 6].

The Type I seesaw[8]) mechanism offers a more attractive explanation for small neutrino(Majorana) masses $\left(m_{v} \sim\left(m_{v}^{D}\right)^{2} / M_{v^{c}}\right)$ based on large right handed neutrino masses $M_{v^{c}}>>M_{S}$. It is natural to ask if theories with supersymmetric Type I seesaw masses also support inflation. The popular Leptogenesis[9] scenario as well as the realistic Susy Minimal SO(10)GUT strongly hint at right handed neutrino masses in the range $10^{6}$ to $10^{12} \mathrm{GeV}$. So for $V_{B-L} \sim M_{X}>10^{16} \mathrm{GeV}$ the superpotential couplings $f_{A}, A=1,2,3$ which generate $M_{v_{A}^{c}} \sim f_{A} V_{B-L}$, are very small $\left(f_{A} \sim 10^{-9}\right.$ to $\left.10^{-4}\right)$ and can also give rise to a cubic term in the quartic inflaton potential. Thus the required ingredients for inflation are already present in Supersymmetric Type I seesaw models. 
Issues regarding natural values for superpotential couplings come into focus when viewed in the context of the so called Minimal Left Right supersymmetric models[10] and their embedding in GUT models[11, 12]. SUSY LeftRight Models are advantaged due to their protection of R-parity as a gauged discrete symmetry, which provides a stable lightest supersymmetric particle (LSP) which has the properties required to serve as WIMP dark matter. They simultaneously and naturally implement Seesaw mechanisms for neutrino masses[10]. Moreover such models have also been incorporated in the realistic and predictive New Minimal Susy SO(10) grand unified theories(NMSGUT)[13, 14] where all the hard parameters of the MSSM are fitted in terms of fundamental parameters of the GUT and soft SUSY breaking parameters (of the Non-Universal Higgs masses (NUHM) type) defined at the Unification scale $M_{X} \sim 10^{16}-10^{18} \mathrm{GeV}$. Such GUTs have viable Bino dark matter candidates and make distinctive predictions for the type of SUSY spectra observable at the LHC. In 2008, well before the discovery of Higgs mass of around $125 \mathrm{GeV}$ in 2011-2012 and the consequent realization that a general framework such as the phenomenological MSSM (pMSSM) requires that the soft trilinear couplings $A_{t, b}$ be large, we concluded[13] that the NMSGUT would be falsified by its failure to fit the down type quark masses unless $A_{0}, \mu$ were in the tens/hundreds of $\mathrm{TeV}$ : leading to a mostly decoupled mini-split supersymmetry type superspectrum with only the LSP, gauginos and possibly a light slepton in the sub-TeV range. The experimental data has now forced this realization on practitioners of MSSM parametrology[15]. In the NMSGUT it was a prediction.

Taken together with the possibility of small values for the light generation Yukawa Dirac couplings it is possible to implement viable inflection point inflation by suitable tuning at the supersymmetric level itself. This is technically more appealing than a tuning applied to soft susy parameters which, being unprotected by SUSY, are unstable. We examine the reheating dynamics briefly. We then derive derive the embedding of SSI in the NMSO(10)GUT and the necessary tuning conditions and show how to satisfy them explicitly.

\section{GENERIC PROPERTIES OF RENORMALIZABLE INFLECTION POINT INFLATION}

In this section we outline the essential features of inflection point inflation deriving from a quartic potential of a single complex scalar field $\varphi$. Since the angular degree of freedom has positive curvature and cannot support inflation one may assumed it fixed at its minimum by relaxation and focus on the remaining real field $\phi$ whose potential is generically $V=\frac{h^{2}}{12} \phi^{4}-\frac{A h}{6 \sqrt{3}} \phi^{3}+\frac{M^{2}}{2} \phi^{2}$.

The fine-tuning $A=4 M$ implies that one has a saddle point $\left(V^{\prime}\left(\phi_{0}\right)=V^{\prime \prime}\left(\phi_{0}\right)=0\right)$ at the field value $\phi_{0}=\frac{\sqrt{3} M}{h}$. So one defines fine-tuning parameter $\Delta$ through $A=4 M \sqrt{1-\Delta}\left(\Delta=\beta^{2} / 4\right.$ in the notation of [6]). The inflection $\operatorname{point}\left(V^{\prime \prime}\left(\phi_{0}\right)=0\right)$ is at $\phi_{0}=\frac{\sqrt{3} M}{h}\left(1-\Delta+O\left(\Delta^{2}\right)\right)$. For small $\Delta$

$$
V\left(\phi_{0}\right)=V_{0}=\frac{M^{4}}{4 h^{2}}(1+4 \Delta) \quad ; \quad V^{\prime}\left(\phi_{0}\right)=\alpha=\frac{\sqrt{3} M^{3} \Delta}{h} \quad ; \quad V^{\prime \prime \prime}\left(\phi_{0}\right)=\gamma=\frac{2 M h}{\sqrt{3}}(1-2 \Delta)
$$

If $h$ is tiny $V_{0}>>M^{4}$ and $\phi_{0}>>M / h ; \gamma$ is small with $h$, while $\alpha$ is small by tuning. Large vacuum energy and flatness around $\phi_{0}$ and starting $\phi$ near $\phi_{0}$ with $\dot{\phi}<<\phi_{0}^{2}$ imply the universe executes slow roll inflation as $\phi$ rolls through an interval of width $\Delta \phi \sim V_{0} / \gamma M_{p}^{2}$ below $\phi_{0}$. The standard slow roll parameters are defined as $\left(M_{p}=2.43 \times 10^{18} \mathrm{GeV}\right)$

$$
\eta(\phi)=\frac{M_{p}^{2} V^{\prime \prime}}{V} \simeq \frac{M_{p}^{2}}{V_{0}} \gamma\left(\phi-\phi_{0}\right) ; \varepsilon(\phi)=\frac{M_{p}^{2}}{2}\left(\frac{V^{\prime}}{V}\right)^{2} \simeq\left(\alpha+\frac{\gamma}{2}\left(\phi-\phi_{0}\right)^{2}\right)^{2}\left(\frac{M_{p}^{2}}{2 V_{0}^{2}}\right) ; \xi=\frac{M_{p}^{4} V^{\prime} V^{\prime \prime \prime}}{V^{2}} \simeq \frac{M_{p}^{4} \alpha \gamma}{V_{0}^{2}}
$$

The observed CMB is a combined spectrum of modes which exited the horizon during inflation. We approximate it as a single spectrum from a representative("pivot") mode that exits the co-moving horizon when $\phi=\phi_{C M B}$. This is the field value near $\phi_{0}$ where the inflation giving rise to observable effects today kicks in (when $N_{C M B}$ e-folds of inflation are remaining). The power spectrum and spectral index we see today are then $P_{R}\left(\phi\left(N_{\mathrm{CMB}}\right)\right)$ and $n_{s}\left(\phi\left(N_{\mathrm{CMB}}\right)\right)$ respectively. The small first and third Taylor coefficients $\alpha, \gamma$ determine[7, 16, 17] the measured parameters of inflation $\left(P_{R}, n_{s}\right)$ once the field values $\left(\phi_{C M B}, \phi_{\text {end }}\right)$ at the time of horizon entry of the "pivot" momentum scale $\left(k_{\text {pivot }}=0.002 \mathrm{Mpc}^{-1}\right)$ and at termination of the slow roll are fixed[7, 17](on the basis of an overall cosmogonic scenario and the consistency of the slow roll approximation $\left(\eta\left(\phi_{\text {end }}\right) \approx 1\right)$ respectively). The observable number of efolds $N_{C M B}=N\left(\phi_{C M B}\right)$ is the number of e-folds of inflation left to occur after $\phi$ crosses $\phi_{C M B}$ (the field value when the representative primordial fluctuation length scale $\left(l_{\text {pivot }}=k_{\text {pivot }}^{-1}\right)$ becomes larger than the comoving horizon $\left.\left(1 / a_{k} H_{k}\right)\right)$. Plausible inflationary cosmogonies require $40<N_{C M B}<60$ and this severely restricts the inflation exponents. 
The slow roll inflation formula for the power spectrum of the mode that is leaving the horizon when the inflaton rolls to $\phi$ and the corresponding spectral index and it's variation with momentum is([16])

$$
P_{R}(\phi)=\frac{V_{0}}{24 \pi^{2} M_{\mathrm{p}}^{4} \varepsilon(\phi)} ; n_{s}(\phi) \equiv 1+2 \eta(\phi)-6 \varepsilon(\phi) ; \mathscr{D}_{k}\left(n_{s}\right)=\frac{k d n_{s}(\phi)}{d k}=-16 \varepsilon \eta+24 \varepsilon^{2}+2 \xi^{2}
$$

In practice $\varepsilon, \xi$ are so small in the narrow region near $\phi_{0}$ where slow-roll inflation occurs that their contribution to $n_{s}$ is negligible. $\mathscr{D}_{k}\left(n_{s}\right)$ is negligible i.e. the spectral index is scale invariant in the observed range, as is allowed by observation so far.

The field value at the end of inflation $\phi_{\text {end }}$ is defined by $\eta\left(\phi_{\text {end }}\right) \simeq 1$ which gives $\phi_{0}-\phi_{\text {end }}=\frac{V_{0}}{\gamma M_{p}^{2}}$. In the slow roll approximation $\dot{\phi}=-V^{\prime}(\phi) / 3 H>>\ddot{\phi} / H$, where $H=\sqrt{V\left(\phi_{0}\right) /\left(3 M_{p}^{2}\right)}$ is the (constant) inflation rate during slow roll inflation. One has the $N-\phi$ link (which can be exactly inverted[28], without assuming that $\phi_{\text {end }}<<\phi(N)$ [7])):

$$
N(\phi)=-3 \int_{\phi}^{\phi_{\text {end }}} \frac{H^{2}}{V^{\prime}(\phi)} d \phi=\sqrt{\frac{2}{\alpha \gamma}} \frac{V_{0}}{M_{p}^{2}}\left(\arctan \sqrt{\frac{\gamma}{2 \alpha}}\left(\phi_{0}-\phi_{\text {end }}\right)-\arctan \sqrt{\frac{\gamma}{2 \alpha}}\left(\phi_{0}-\phi\right)\right)
$$

$N_{\text {pivot }}$ is estimated using the standard Big Bang thermal cosmogony. giving [17] $N_{\text {pivot }}=65.5+\ln \frac{\rho_{r h}^{\frac{1}{12}} V_{0}^{\frac{1}{6}}}{M_{\mathrm{p}}}$ where $\rho_{r h}$ is the energy density after reheating and $V_{0}$ the potential value during inflation. Due to rapid thermalization in this model(see below) the two are equal and then since the scale is set by $V^{1 / 4} \sim M / \sqrt{h}$ one finds $N_{\text {pivot }}=46$ to $55=51 \pm 5$ to be a reasonable estimate.

To search for sets of potential parameters $M, h, \Delta$ compatible with the observed $P_{R}, n_{S}, N_{C M B}$ one uses the definitions

$$
\varepsilon_{C M B}=\frac{V_{0}}{24 \pi^{2} M_{p}^{4} P_{R}} \quad ; \quad \eta_{C M B}=\frac{\left(n_{s}-1\right)}{2}
$$

and from these deduces $\alpha_{C M B}, \phi_{C M B}$ using the eqns.(2)

$$
\phi_{C M B}=\phi_{0}+\frac{V_{0} \eta_{C M B}}{\gamma M_{p}^{2}} \quad ; \quad \alpha_{C M B}=\sqrt{2 \varepsilon_{C M B}} \frac{V_{0}}{M_{p}}-\frac{V_{0}^{2} \eta_{C M B}^{2}}{2 \gamma M_{p}^{4}}
$$

The required fine-tuning $\Delta$ is then

$$
\Delta=\frac{h \alpha_{C M B}}{\sqrt{3} M^{3}}=\left(\frac{M}{4 h M_{p}}\right)^{4}\left(\frac{16 h^{2} M_{p}}{3 \pi M \sqrt{P_{R}}}-\left(1-n_{s}\right)^{2}\right)
$$

$\alpha_{C M B}, \Delta$ should emerge real and positive and using $\left\{\alpha_{C M B}, \phi_{C M B}\right\}$ in the formula for $N_{C M B}$ one should obtain a sensible value in the range $N_{C M B}=51 \pm 5$. Using eqns.(1,5) in eqn(4) we can solve accurately for the required relation between $h, m, \Delta$ using an interpolating function [28]. The result is that $N_{C M B} \sim 50, Z_{0} \approx \frac{1.2}{N_{C M B}}$ solves the exact equations to a good approximation and one obtains the generic constraints :

$$
\frac{h^{2}}{M} \approx \frac{3 \pi}{M_{P}} \frac{\sqrt{P_{R}}}{N_{C M B}^{2}} \approx \frac{2.75 \times 10^{-22}}{N_{C M B}^{2}} \approx 10^{-25} \mathrm{GeV}^{-1} \quad ; \quad \frac{\Delta}{M^{2}} \approx \frac{4.14 \times 10^{-34}}{N_{C M B}^{2} P_{R}} \approx 10^{-28.2} \mathrm{GeV}^{-2}
$$

We then have viable inflation with inflaton energy and Hubble rate

$$
V_{0} \sim \frac{M^{4}}{h^{2}} \sim(M)^{3} \times 10^{25} \mathrm{GeV} \sim 10^{43}-10^{61} \mathrm{GeV}^{4} \quad ; \quad H_{0} \sim \sqrt{\frac{V_{0}}{M_{P}^{2}}} \sim 10^{3}-10^{12} \mathrm{GeV}
$$

The fine-tuning measure grows as $M^{2}$ so that $\beta=\sqrt{\Delta}$ can be as large as $10^{-2}$ for $M \sim 10^{12} \mathrm{GeV}$. In our scenario due to the large value of the inflaton mass parameter $M \sim 10^{6}$ to $10^{13} \mathrm{GeV}$ compared to $M \sim T e V$ in the case of MSSM inflation [3] or Dirac neutrino inflation [5, 7] the fine-tuning required is quite mild and removes much of the motivation for complicated just so hybrid inflation scenarios.

We can also estimate the ratio $r$ of power in Tensor and Scalar CMB fluctuations using $r=2 V_{0} /\left(3 \pi^{2} P_{R} M_{P}^{4}\right)$. On using eqn.(8)

$$
r=\left(\frac{M}{7.95 \times 10^{13} \mathrm{GeV}}\right)^{3}
$$

This makes the observation of tensor perturbations in such a scenario hard unless $M$ is near its upper limit. 


\section{SUPERSYMMETRIC SEESAW INFLATON MODEL}

In this section we introduce a toy one generation Supersymmetric seesaw inflation scenario model with gauge group $S U(3) \times S U(2) \times U(1)_{R} \times U(1)_{B-L}$ that captures the essential features of our scenario. The essential fields beyond the MSSM are a right handed Neutrino chiral multiplet $N[1,1,-1 / 2,1]$ and a field $S[1,1,1,-2]$ whose vev generates the large Majorana masses $M_{V}\left(10^{6}-10^{14} \mathrm{GeV}\right)$ for the conjugate neutrinos $v_{A}^{c} \equiv N_{A}$ via a renormalizable superpotential coupling $3 \sqrt{2} f_{A B} S v_{A}^{c} v_{B}^{c}$. Additional superheavy fields $\Omega_{i}$ serve to fix the vev of $S$ as in Minimal Supersymmetric Left Right Models (MSLRMs)[10] and in GUTs that embed them [11,12,13]. Neutrino Dirac Yukawa coupling is present in the superpotential : $W=y_{v} N L H+\ldots$ where $L[1,2,0,-1], H[1,2,1 / 2,0]$ are the Lepton doublet and up type Higgs respectively. The relevant $\mathrm{D}$-flat direction extends out of the minimum of the supersymmetric potential corresponding to the breaking of the gauge group down to the MSSM symmetry

$$
S U(3) \times S U(2) \times U(1)_{R} \times U(1)_{B-L} \rightarrow S U(3) \times S U(2) \times U(1)_{Y}
$$

This leads to a Type I seesaw plus MSSM (SIMSSM) effective theory. After symmetry breaking the MSSM hypercharge $Y=2 T_{3 R}+(B-L)$ where $T_{3 R}$ is the $U(1)_{R}$ generator. Unlike the case of the Dirac neutrino masses scenario [5] $B-L$ is not a gauge symmetry down to low energies. This can have important consequences for nucleosynthesis and matter domination since the heavy right handed neutrinos must find a non-gauge channel to decay through. The flat-direction associated with the gauge invariant $N L H$ is then specified as

$$
\tilde{N}=\tilde{v}=h_{0}=\frac{\varphi}{\sqrt{3}}=\phi e^{i \theta} ; \quad \phi \geq 0, \quad \theta \in[0,2 \pi)
$$

The additional fields $\Omega_{i}$ are coupled to $S$ so that extremization of the SUSY potential using $F_{\Omega_{i}}=0,\left.D_{\alpha}\right|_{\phi=0}=0$ fixes the vev of $\mathrm{S}:\langle S\rangle=\bar{\sigma} / \sqrt{2}$ without constraining the inflaton field $\varphi$. This is as in the Minimal Susy LR models[10] and renormalizable Susy SO(10) GUTs $[12,13]$ which are our inspiration and target.

At scales $\phi \sim \bar{\sigma}>>M_{S}$ where SUSY is exact the relevant superpotential is given by:

$$
W=3 \sqrt{3} y N v h+3 f \sqrt{2} S N N+\ldots=y \varphi^{3}+f \sqrt{2} S \varphi^{2}+\ldots
$$

where $h, f, \bar{\sigma}$ can be taken real without loss of generality.The equations of motion of the unperturbed vacuum imply $<F_{S}>=0,<S>=\bar{\sigma} / \sqrt{2}$. The right handed neutrino Majorana mass will be $M_{v^{c}}=6 f \bar{\sigma}$.

This superpotential leads to an inflaton potential

$$
\begin{aligned}
V_{\text {susy }} & =\left|3 y \varphi^{2}+2 f \bar{\sigma} \varphi\right|^{2}+2\left|f \varphi^{2}\right|^{2} \\
& =f^{2}\left[\left(2+9 \tilde{y}^{2}\right) \phi^{4}+12 \tilde{y} \phi^{3} \bar{\sigma} \cos \theta+4 \bar{\sigma}^{2} \phi^{2}\right]
\end{aligned}
$$

Here $\tilde{y}=y / f$ and $f \bar{\sigma}$ sets the mass scale. Minimizing with respect to $\theta$ gives $\theta=\pi$ so we can focus on just the real part of $\varphi$ and set $\varphi=-\phi$ with $\phi$ real and positive near the inflection point but free to fall into the well around $\phi=0$ and oscillate around that value. In addition one also expects a contribution to the potential from the $\mu$ term for the Higgs doublets together with SUSY breaking quadratic and cubic soft terms, which we assume to be of the type generated by supergravity, but with non universal Higgs masses, i.e of the form:

$$
\begin{aligned}
V_{\text {soft }} & =\left[A_{0}\left(y \varphi^{3}+f \sqrt{2} S \varphi^{2}\right)+h . c\right]+m_{\tilde{f}}^{2} \sum_{\tilde{f}}|\tilde{f}|^{2}+m_{H}^{2}|H|^{2}+m_{\bar{H}}^{2}|\bar{H}|^{2} \\
& =f^{2}\left[\tilde{y} \tilde{A}_{0} \phi^{3} \bar{\sigma} \cos 3 \theta+\tilde{A}_{0} \bar{\sigma}^{2} \phi^{2} \cos 2 \theta+\tilde{m}_{0}^{2} \bar{\sigma}^{2} \phi^{2}\right]
\end{aligned}
$$

here $\tilde{m}_{0}=m_{0} / f \bar{\sigma}, \tilde{A}_{0}=2 A_{0} / f \bar{\sigma}$. The soft mass $m_{0}$ receives contributions from the sfermion and Higgs soft masses as well as the $\mu$ term : $m_{0}^{2}=\left(2 m_{\tilde{f}}^{2}+\bar{m}_{H}^{2}\right) / 3$. Here $m_{\tilde{f}, H}$ are the sfermion and up type Higgs soft effective masses at the unification scale $\left(\bar{m}_{H}^{2}=m_{H}^{2}+|\mu|^{2}\right)$. Since these masses and $A_{0}$ should be in the range $10^{2}-10^{5} \mathrm{GeV}$ while the righthanded neutrino masses lie in the range $10^{6}-10^{12} \mathrm{GeV}$, it is clear that $\tilde{m}_{0}, \tilde{A}_{0}$ are small parameters and even for the large values of $m_{0}, A_{0} \sim 10^{5} \mathrm{GeV}$ found in the NMSGUT $\tilde{m}_{0}, \tilde{A}_{0}<<1$. Thus these terms cannot significantly change $\theta=\pi$ assumed earlier. The total inflaton potential is then

$$
V_{t o t}=f^{2}\left(\left(2+9 \tilde{y}^{2}\right) \phi^{4}-\left(\tilde{A}_{0}+12\right) \tilde{y} \bar{\sigma} \phi^{3}+\left(\tilde{A}_{0}+\tilde{m}_{0}^{2}+4\right) \bar{\sigma}^{2} \phi^{2}\right) .
$$


Thus we have a generic quartic inflaton potential of the same type as in Section $\mathbf{2}$ but the parameter values in the case of Type I seesaw are quite different from the light Dirac neutrino case. We have the identification of parameters

$$
\begin{aligned}
h & =f \sqrt{12\left(2+9 \tilde{y}^{2}\right)} \\
A & =\frac{3 f\left(\tilde{A}_{0}+12\right) \tilde{y} \bar{\sigma}}{\sqrt{\left(2+9 \tilde{y}^{2}\right)}} \\
M^{2} & =2 f^{2} \bar{\sigma}^{2}\left(4+\tilde{A}_{0}+\tilde{m}_{0}^{2}\right) \\
\Delta & =\left(1-\frac{A^{2}}{16 M^{2}}\right) \\
& =\left(1-\frac{9 \tilde{y}^{2}\left(\tilde{A}_{0}+12\right)^{2}}{32\left(2+9 \tilde{y}^{2}\right)\left(\tilde{A}_{0}+\tilde{m}_{0}^{2}+4\right)}\right)
\end{aligned}
$$

For seesaw models the natural magnitude for the neutrino Dirac mass is, $m_{v}^{D}>1 \mathrm{MeV}$ (i.e $\left|y_{v}^{D}\right|>10^{-5}$ and then the limit $m_{v}<<0.01 \mathrm{eV}$ for the lightest neutrino (assuming direct hierarchy) implies $M_{v^{c}}>10^{6} \mathrm{GeV}$ ). Since the preferred values for the Susy breaking scale are smaller than $100 \mathrm{TeV}$ (at most) it follows that the maximum value of $\left|\tilde{A}_{0}\right|,\left|\tilde{m}_{0}\right| \sim 0.1$ and they could be much smaller for more typical larger values of the conjugate neutrino masses $M_{V^{c}} \sim 10^{8}$ to $10^{12} \mathrm{GeV}$. It is then clear from the corresponding range $\Delta \sim 10^{-12}$ to $10^{-4}$ that the coupling ratio $\tilde{y}=y / f$ becomes ever closer to exactly $\tilde{y}=4 / 3$ as $M$ increases and even for $M \sim 10^{6} \mathrm{GeV}$ differs from 1.333 only at the second decimal place. Thus to a good approximation $h=6 \sqrt{6} f$. Then it follows that

$$
f \simeq 10^{-26.83 \pm 0.17}\left(\frac{\bar{\sigma}}{G e V}\right) ; M \simeq 10^{-25.38 \pm 0.17}\left(\frac{\bar{\sigma}}{G e V}\right)^{2} ; \Delta \simeq 10^{-78.93 \pm 0.47}\left(\frac{\bar{\sigma}}{G e V}\right)^{4}
$$

The range $M \sim 10^{6.6}$ to $10^{10.6} \mathrm{GeV}$ corresponds nicely to $10^{16} \mathrm{GeV}<\bar{\sigma}<10^{18} \mathrm{GeV}$ : as is natural in single scale Susy SO(10) GUTs[11, 12, 13, 14]. $f$ increases with $\bar{\sigma}$ with values below $10^{-11}$ achievable in the NMSGUT only with difficulty. In MSLRMs, since there are no GUT constraints on $\bar{\sigma}$, one may assume somewhat wider ranges for these parameters.

In all relevant cases $\Delta<10^{-4}$ is required. Thus the above equations imply that $\tilde{y}^{2}$ must be close to the value

$$
\tilde{y}_{0}^{2}=\frac{64}{9} \frac{4+\tilde{A}_{0}+\tilde{m}_{0}^{2}}{16-8 \tilde{A}_{0}-32 \tilde{m}_{0}^{2}+\tilde{A}_{0}^{2}}
$$

Here $\tilde{A}_{0}, \tilde{m}_{0} \sim O\left(M_{S} / M_{v^{c}}\right)<<1$, hence $\tilde{y}_{0}$ is rather close to $4 / 3$ and the equality is very close for larger $M \sim f \bar{\sigma}$ since then $\tilde{A}_{0}, \tilde{m}_{0}$ are tiny. The measure of severity of fine tuning $\beta=\sqrt{\Delta} \sim 10^{-2}-10^{-6}$ compares quite favourably with the case of the MSSM or Dirac neutrino inflaton since there $\beta \sim 10^{-12}$ to $10^{-10}$ due to the low values of the inflaton mass in those cases. The dominant component of the fine tuning in the present case is a fine-tuning of superpotential parameters, which is radiatively stable due to non renormalization theorems. Specially for large $\bar{\sigma}>10^{16} \mathrm{GeV}$ the Type I Susy seesaw can provide a rather attractive inflationary seesaw with a natural explanation for neutrino masses and weaker tuning demands on the radiatively unstable Susy breaking parameters than the extreme and unstable finetunings demanded by typical inflection point scenarios and in particular the Dirac neutrino model [5]. Moreover, unlike the chaotic sneutrino inflaton scenario[18, 19], no trans-Planckian vevs are invoked.

\section{Reheating and Leptogenesis}

The post inflationary dynamics of our model bears an intimate relation to previous studies of models with 'instant preheating' mechnism[20] and specially the MSSM flat direction inflection point inflation model[21] and preheating model[22] with strong coupling to the MSSM Higgs. Supersymmetric seesaw inflation offers an attractive synthesis precisely fulfilling the need expressed in [22] :

"There have been many models of leptogenesis. A hallmark of our model is the economy of fields. The only undiscovered fields are the inflaton, $\phi$, the standard model Higgs, $h$, and the right-handed neutrino, $N$. There are very good reasons for suspecting that all exist! The only unfamiliar aspect of our model is the strong coupling of the 
inflaton field to the Higgs field. While there is no reason to preclude such a coupling, it would be very interesting to find particle-physics models with a motivation for the coupling. "

Due to the gauge $(\mathrm{H}, \mathrm{L})$ and third generation yukawa $(H)$ coupled components of the inflaton the inflaton energy will decay very rapidly (with decay time $\tau_{d e c}<<H_{\text {infl }}^{-1} \sim\left(h M_{p}\right) / M^{2}$ ) by the "instant preheating" mechanism[20, 22, 21]. Thus the reheating temperature $T_{r h} \sim T_{\max } \sim V_{0}^{1 / 4} \sim M / h^{1 / 2} \sim 10^{11}-10^{15} \mathrm{GeV}$. The parametric dependence is identical to that found in [21]. The difference in scales arises only because the inflaton mass $M \sim 10^{6}-10^{12} \mathrm{GeV}$ in our model is much larger than the inflaton mass parameter $m_{\phi} \sim 0.1-10 \mathrm{TeV}$ in [21] coming from soft Supersymmetry breaking.

In preheating (" $\chi$ type") degrees of freedom, with masses $\left(m_{\chi} \sim g \phi(t)\right)$ and decay rates $\left(\Gamma \sim g^{3} \phi(t)\right)$ proportional to $\phi(t)$, are produced non-perturbatively every time the inflaton field crosses zero. This happens because the $\chi$ modes are ultra-light for a sufficiently large time interval around the zero crossing time during which adiabaticity is violated ( $\dot{\omega}_{k}>\omega_{k}^{2}$ : where $\omega_{k}$ is the oscillation frequency at wave number $k$ ). Here $\chi$ modes are the components of the $H, L, u_{L}^{c}, u_{L}$ chiral superfields and the $W_{ \pm}, B$ gauge superfields. They can be identified as the fields which become massive given background values of the three components of the inflaton $\left(\tilde{v}, \tilde{v}_{L}^{c}, h^{0}\right)$. Then with the usual superpotential

$$
W=y^{u} Q_{L} H u_{L}^{c}+y^{d} Q_{L} \bar{H} d_{L}^{c}+y^{v} L H N+y^{l} L \bar{H} e_{L}^{c}+\ldots
$$

$y^{u}$ leads to massive $u_{L}, u_{L}^{c} ; y^{v}$ leads to massive $e_{L}$ (one combination of the three $e_{L}$ ), $h^{0}, h^{+}, v_{L}, v_{L}^{c} ; y^{l}$ leads to massive $\bar{h}^{-}, e_{L}^{c}$ (one combination). Since $\langle H, N, L\rangle$ preserve $U(1)_{e m}$, the gauge couplings give masses to $\mathrm{Z}$ (which forms a Dirac supermultiplet with $\left(v-\tilde{h}_{0}\right) / \sqrt{2}$ ) and $W_{ \pm}$(form a pair of Dirac supermultiplets with $l^{-}, h_{+}$). The inflaton vev leaves the down quark and gluon/gluino fields and $\bar{h}_{0}$, and some combinations of the $l_{L}^{-}, l_{L}^{c}$ fields with light (MSSM type) masses. These light ( $\psi$-type) fields will form the first step in the decays of the $\chi$ field. As $\langle\phi\rangle$ again increases after crossing zero the $\chi$ modes become heavy and unstable and as a result decay rapidly(within a time $\tau_{d e c} \sim \frac{h}{M g^{3}}<<m_{\phi}^{-1}$ ) to the light (mostly coloured) MSSM modes to which they are coupled strongly coupled. A fraction $\sim 10^{-1}$ of the inflaton condensate energy passes into the light MSSM modes with every crossing resulting in complete transfer within $\sim 10^{2}$ oscillation times. $\tau_{\text {osc }} \sim m_{\phi}^{-1}<<H_{\text {infln }}^{-1} \sim\left(h M_{p}\right) \tau_{o s c} / M \sim(1-150) \tau_{\text {osc }}$. Once the energy is in the light $(\psi)$ modes MSSM interactions rapidly complete thermalization. Rapid decay of the inflaton oscillation amplitude leaves the light modes to thermalize the energy dumped by the inflaton into a radiation bath of all modes: which are no longer ever heavy because the inflaton has decayed. The reheating temperature is

$$
T_{r h} \sim\left(\frac{30}{\pi^{2} g_{*}}\right)^{1 / 4} V_{0}^{1 / 4} \sim T_{\max } \sim 10^{11}-10^{15} \mathrm{GeV}
$$

where $g_{*}=228.75$ is the effective number of MSSM degrees of freedom. This reheating temperature is well above that required to produce relativistic populations of gravitinos : which are unacceptable if their lifetimes are larger than the nucleosynthesis time $\tau_{N} \sim 1 \sec$ since their decay after nucleosynthesis would destroy the created nucleons. The straightforward and generic resolution of this gravitino problem is if the graviton masses are sufficiently large so that the gravitinos decay before nucleosynthesis[23]: $\tau_{\text {grav }} \sim 10^{5} \sec \left(\frac{1 \mathrm{TeV}}{m_{3 / 2}}\right)^{3}<<\tau_{N} \sim 1 \mathrm{sec}$. Thus Supersymmetric seesaw Inflation also indicates that the scale of supersymmetry breaking -as indicated by the gravitino mass- should be above $50 \mathrm{TeV}$; as is also found by fitting of fermion data in the NMSGUT[13]. Large reheat temperatures also ensure abundant thermal production of righthanded neutrinos after inflation. Their CP violating decays into leptons can drive thermal lepto-genesis [9] for generating the observed baryon to entropy density $n_{B} / s \sim 10^{-10}$. Non-thermal leptogenesis is also possible [22] since the Higgs field $\mathrm{H}$ is itself a $\chi$ type field and coupled to the righthanded neutrinos. During inflaton oscillations the Higgs mass $m_{h} \sim g_{2} \phi$ fluctuates below and above $M_{v^{c}} \sim f \bar{\sigma}$. CP violating Higgs-righthanded Neutrino inter-conversion[22] leads to (non-thermal) Leptogenesis which will add to the thermal leptogenesis. The complication in the present case that the $L, H$ and $N$ components of the inflaton have different decay rates implies a proper analysis must track the separate evolution of all three fields making up the inflaton using the equation of motion and Boltzmann equation for the relevant degrees of freedom. This requires a separate numerical study to expose the interplay of the couplings $f_{A}, y_{A B}, g_{2}$. The study of this evolution and the operation of Leptogenesis in these models is now in progress.

\section{INFLATION AND NEUTRINO MASSES IN THE NMSGUT}

Finally we embed SSI in the New Minimal SO(10) GUT (NMSO(10)GUT or NMSGUT). The NMSGUT is a realistic Susy SO(10) model[13, 14, 25] that successfully fits the known fermion mass-mixing data in terms of GUT parameters 
and provides structural reasons for suppression of the dangerous operator dimension $d=4,5$ Baryon violation typical in Susy GUTs[14]. It furthermore makes distinctive predictions of a mini-split supersymmetry spectrum made viable by large $A, \mu$-terms and with a characteristic normal s-hierarchy. Neutrino flavour plays a key role in enabling NMSGUT inflation : the inflaton is composed of third generation conjugate sneutrino, first generation left slepton (sneutrino) and the $T_{3 R}=1 / 2$ Higgs.

The NMSGUT Higgs field vevs $\{\mathbf{2 1 0}(\omega, p, a), \mathbf{1 2 6}(\sigma)\} \equiv \Omega, \overline{\mathbf{1 2 6}}(S=\bar{\sigma})$ break $S O(10) \rightarrow G_{M S S M}$ while preserving Supersymmetry at $M_{X}$. An explicit Susy preserving solution of symmetry breaking in terms of a cubic equation for a complex variable $x$ and depending on a single parameter ratio $\xi$ was found by us[12]. The mass spectra implied[12,13] by this analytic solution for the the MSGUT vacuum are the basis of our detailed Renormalization Group and threshold effect analysis $[12,13,14]$. Inclusion of threshold corrections raises the unification scale close to the Planck scale and can lower the gauge coupling at unification. We shall use the notation and results of [26, 27, 13, 14].

To embed SSI corresponding to a NLH type flat direction we show there is a corresponding flat direction of the full GUT potential which rolls out of the MSGUT minimum (that has the SIMSSM as its effective theory). The relevant fields are the GUT scale vev fields $\Omega \equiv\{\omega, p, a, w, \sigma\}, S=\bar{\sigma}$ and the (6) possible components $h_{i}, \bar{h}_{i} ; i=1 \ldots 6$ of the light MSSM Higgs doublet pair $H, \bar{H}$ together with the chiral lepton fields $L_{A}, v_{A}^{c}, A=1,2,3$. The relevant superpotential is then $[12,13]$

$$
W=2 \sqrt{2}\left(h_{A B} h_{1}-2 \sqrt{3} f_{A B} h_{2}-g_{A B}\left(h_{5}+i \sqrt{3} h_{6}\right)\right)+\bar{h}^{T} \mathscr{H}(<\Omega>) h+4 \sqrt{2} f_{A B} \bar{\sigma} \bar{v}_{A} \bar{v}_{B}+W_{\Omega}(\Omega)
$$

where

$$
W_{\Omega}(\Omega, \bar{\sigma})=m\left(p^{2}+3 a^{2}+6 \omega^{2}\right)+2 \lambda\left(a^{3}+3 p \omega^{2}\right)+(M+\eta(p+3 a-6 \omega)) \sigma \bar{\sigma}
$$

and

$$
\left.\frac{\partial W_{\Omega}}{\partial \Omega}\right|_{h, \bar{v}, L=0}=\left.\frac{\partial W_{\Omega}}{\partial \bar{\sigma}}\right|_{h, \bar{v}, L=0}=\left.0 \quad D_{\alpha}(\Omega)\right|_{h, \bar{v}, L=0}=0
$$

here $h_{A B}, g_{A B}, f_{A B}$ are the yukawa coupling matrices of the three matter 16-plets to the 10,120, $\overline{\mathbf{1 2 6}}$ Higgs multiplets respectively. $\mathscr{H}$ is the Higgs doublet mass matrix[27, 12, 13]. Equation (24) defines the MSGUT vacuum[12].

Of the 5 diagonal D-terms of $\mathrm{SO}(10)$ only those corresponding to the generators $T_{3 L}$, $T_{3 R}, B-L$ are relevant for vevs $\Omega, \bar{\sigma}$ and out of equilibrium inflaton mode composed of $v, v^{c}, h_{0}$. The vevs $\Omega, \bar{\sigma}$ do not contribute to these $\mathrm{D}$ terms or cancel so

$$
\begin{aligned}
D_{3 L} & =\frac{g_{u}}{2}\left(-\sum_{i=1}^{6}\left|h_{i 0}\right|^{2}+\sum_{A}\left|\tilde{v}_{A}\right|^{2}\right) \\
D_{3 R} & =\frac{g_{u}}{2}\left(\sum_{i=1}^{6}\left|h_{i 0}\right|^{2}-2\left|h_{40}\right|^{2}-\sum_{A}\left|\tilde{\bar{v}}_{A}\right|^{2}\right) \\
D_{B-L} & =\sqrt{\frac{3}{8}} g_{u}\left(\sum_{A}\left(\left|\tilde{\bar{v}}_{A}\right|-\left|\tilde{v}_{A}\right|^{2}\right)+2\left|h_{40}\right|^{2}\right)
\end{aligned}
$$

where only $h_{4 \alpha}=\Phi_{2 \alpha}^{44}$ has $B-L=+2, T_{3 R}=-1 / 2$ and thus $Y=1$ while all others have $T_{3 R}=1 / 2$ and $B-L=0$. The D-flatness conditions are

$$
\sum_{A}\left|\tilde{v}_{A}\right|^{2}=\sum_{i}\left|h_{i 0}^{2}\right|=\sum_{A}\left|\tilde{\bar{v}}_{A}\right|^{2}+2\left|h_{40}\right|^{2}
$$

In MSGUTs the MSSM Higgs doublet pair is defined by fine tuning $\operatorname{Det}(\mathscr{H}) \simeq 0$ so that its lightest eigenvalue $\mu \sim M_{W} \sim 1 \mathrm{TeV}$ specifies the $\mu$ term in the superpotential of the SIMSSM $: W=\mu \bar{H} H+\ldots$. The doublet pair $H, \bar{H}$ is a linear combination $[12,27,26]$ of the 6 doublet pairs of the the NMSGUT :

$$
h_{i}=U_{i j} H_{j} \quad \bar{h}_{i}=\bar{U}_{i j} \bar{H}_{j}
$$

where $U, \bar{U}$ diagonalize the doublet mass matrix $\mathscr{H}: \bar{U}^{T} \mathscr{H} U=\operatorname{Diag}\left\{\mu, M_{2}^{H}, \ldots, M_{6}^{H}\right\}$ to positive masses. They are calculated with $\mu=0=\operatorname{Det}(\mathscr{H})$. The so called Higgs fractions : $\alpha_{i}=U_{i 1}, \bar{\alpha}_{i}=\bar{U}_{i 1}$, determine the grand unified formulae[12, 13] for the SIMSSM fermion yukawas. For tree level yukawa couplings replace $h_{i}, \bar{h}_{i} \rightarrow \alpha_{i} H, \bar{\alpha}_{i} \bar{H}$. For example the neutrino Dirac coupling is $\left(\left(\tilde{h}_{A B}, \tilde{g}_{A B}, \tilde{f}_{A B}\right)=2 \sqrt{2}\left(h_{A B}, g_{A B}, f_{A B}\right)\right)$

$$
y_{A B}^{v}=\tilde{h}_{A B} \alpha_{1}-2 \sqrt{3} \tilde{f}_{A B} \alpha_{2}-\tilde{g}_{A B}\left(\alpha_{5}+i \sqrt{3} \alpha_{6}\right)
$$


From the $V=\left|F_{\bar{h}}\right|^{2}$ only the light Higgs doublet $H$ can contribute. To get small yukawas the involvement of the lightest generation is unavoidable. Thus we take $v_{A}=v_{1}$. Taking $\tilde{\tilde{v}}_{A}=\tilde{\tilde{v}}_{1}$ the tuning constraint is at best of form $\left|y_{11}\right|^{2} \sim 10\left(\left|y_{21}\right|^{2}+\left|y_{31}\right|^{2}\right)$ : this is impossible to satisfy with normal neutrino yukawa coupling hierarchy. Choosing $\bar{v}_{A}=v_{3}^{c}$ as the conjugate neutrino component of the inflaton is more helpful in satisfying the fine tuning condition. Thus our inflaton ansatz is

$$
\tilde{v}_{1}=\frac{\phi}{\sqrt{3}} \quad h_{i 0}=\frac{\alpha_{i} \phi}{\sqrt{3}} \quad \tilde{\bar{v}}_{3}=\frac{\phi}{\sqrt{3}} \sqrt{1-2\left|\alpha_{4}\right|^{2}}
$$

Note how the Higgs fraction $\alpha_{4}$ enters as $\Gamma=1-2\left|\alpha_{4}\right|^{2}$. It happens the solutions we have found earlier [13] can have $\left|\alpha_{4}\right| \sim 0.5$. It is not inconceivable that $\Gamma \simeq 0$ is achievable without destroying the realistic fermion fits to the fermion data.

We use generic Supergravity(SUGRY)-NUHM generated soft terms in terms of a common trilinear parameter $A_{0}$ but different soft mass parameters $\tilde{m}_{\tilde{f}}^{2}, \tilde{m}_{h_{i}}^{2}$ for the 16 plets and the different Higgs. Repeating the analysis of section 3 with the NMSGUT superpotential and the new ansatz we obtain the parameter identifications

$$
\begin{aligned}
h & \left.=\frac{2}{\sqrt{3}}\left[\left(y^{v \dagger} y^{v}\right)_{11}+\Gamma\left(\left|\tilde{h}_{31}\right|^{2}+4\left|\tilde{g}_{31}\right|^{2}+\left(y^{v} y^{v^{\dagger}}\right)_{33}\right)+4\left|\tilde{f}_{33}\right|^{2} \Gamma^{2}\right)\right]^{\frac{1}{2}} \\
A & =\frac{1}{h}\left(16\left|\tilde{f}_{33}\right|\left|y_{31}^{v}\right||\bar{\sigma}| \sqrt{\Gamma}+4\left|y_{31}^{v}\right| \sqrt{\Gamma} A_{0} \cos \left(3 \theta_{\bar{\sigma}}-2 \theta_{y_{31}^{v}}\right)\right) \\
M^{2} & =\frac{32}{3}\left|\tilde{f}_{33}\right|^{2}|\bar{\sigma}|^{2} \Gamma+\frac{8}{3} A_{0} \tilde{f}_{33}|\bar{\sigma}| \Gamma \cos \left(3 \theta_{\bar{\sigma}}-2 \theta_{y_{31}^{v}}\right)+2 \widehat{m}_{0}^{2}
\end{aligned}
$$

The fine tuning condition $A=4 M$ is now

$$
\left|y_{31}^{v}\right|^{2}=\frac{8 \Lambda_{n}}{9 \Lambda_{d}-8 \Lambda_{n}(1+\Gamma)}\left[\left|y_{11}^{v}\right|^{2}+\left|y_{21}^{v}\right|^{2}+\Gamma\left(\left|\tilde{h}_{31}\right|^{2}+4\left|\tilde{g}_{31}\right|^{2}+\left|y_{32}^{v}\right|^{2}+\left|y_{33}^{v}\right|^{2}\right)+4\left|\tilde{f}_{33}\right|^{2} \Gamma^{2}\right]
$$

Where

$$
\begin{aligned}
& \Lambda_{n}=1+\frac{A_{0}}{4 M_{3}} \cos \left(3 \theta_{\bar{\sigma}}-2 \theta_{y_{31}^{v}}\right)+\frac{3 \widehat{m}_{0}^{2}}{16 M_{3}^{2} \Gamma} \\
& \Lambda_{d}=\left(1+\frac{A_{0}}{4 M_{3}} \operatorname{Cos}\left(3 \theta_{\bar{\sigma}}-2 \theta_{y_{31}^{v}}\right)\right)^{2}
\end{aligned}
$$

and $M_{3}=\tilde{f}_{33}|\bar{\sigma}| M_{\bar{v}}>>M_{S}$ imples $\Lambda_{n, d}$ are both very close to unity. So as before the fine tuning condition is essentially between hard parameters as in GUTs and in sharp contrast to MSSM inflaton models[3]:

$$
\left|y_{31}^{v}\right|^{2}=\frac{8}{1-8 \Gamma}\left(\Gamma\left(\left|\tilde{h}_{31}\right|^{2}+4\left|\tilde{g}_{31}\right|^{2}+\left|y_{32}^{v}\right|^{2}+\left|y_{33}^{v}\right|^{2}\right)+\left|y_{11}^{v}\right|^{2}+\left|y_{21}^{v}\right|^{2}+4\left|\tilde{f}_{33}\right|^{2} \Gamma^{2}\right)
$$

In NMSGUT fits the strong hierarchy $\left|y_{33}\right|>>\left|y_{32}\right|>>\left|y_{31}\right|>>\left|y_{21}\right|>\left|y_{11}\right|$ holds . So one must tune

$$
\Gamma \approx 0 \text { i.e } \quad\left|\alpha_{4}\right| \approx \frac{1}{\sqrt{2}}
$$

to a good accuracy. The MSSM doublet $\mathrm{H}$ is almost exactly $50 \%$ derived from the doublet in the 210 plet ! The yukawa tuning condition is only

$$
\left|y_{31}^{v}\right|^{2}=8\left(\left|y_{11}^{v}\right|^{2}+\left|y_{21}^{v}\right|^{2}\right)
$$

which is easy to enforce in the NMSGUT.

There is an additional demand coming from eqn $(8): h^{2} / M_{3} \sim\left(y^{v \dagger} y^{v}\right)_{11} / M_{3} \sim 10^{-25}$ which is, at first glance, much harder to enforce. However [14] large wave function corrections[29] to the $\operatorname{GUT}\left(Y_{f}^{\text {tree }}\right)$-MSSM $\left(Y_{f}\right)$ yukawa coupling relation due to the circulation of heavy fields within loops on the lines entering the yukawa vertex imply :

$$
Y_{f}=\left(1+\Delta_{\tilde{f}}^{T}\right) \cdot\left(Y_{f}\right)_{\text {tree }} \cdot\left(1+\Delta_{f}\right)\left(1+\Delta_{H^{ \pm}}\right)
$$


TABLE 1. Illustrative example of relevant parameters from an accurate fit of the fermion spectrum in the NMSGUT which is compatible with inflationary scenario. All masses are in GeV. $\chi_{X, Z}$ are the accuracies of the fits to 18 known fermion mass/mixing parameters at $M_{X, Z}$.

\begin{tabular}{|c|c|c|c|}
\hline Parameter & Value & Parameter & Value \\
\hline$\chi_{X}$ & 0.4458 & $M_{h^{0}}$ & 122.99 \\
$\chi_{Z}$ & 0.1426 & $M_{X}$ & $7.08 \times 10^{17}$ \\
$f_{3}$ & $1.066 \times 10^{-3}$ & $f_{1}, f_{2}$ & $2.59 \times 10^{-8}, 4.405 \times 10^{-5}$ \\
$h$ & $2.44 \times 10^{-4}$ & $\Lambda_{n}$ & 0.999999 \\
$M$ & $3.043 \times 10^{11}$ & $\Lambda_{d}$ & 0.999999 \\
$\Gamma$ & $4.343 \times 10^{-5}$ & $\Delta_{\text {tuning }}$ & 0.989 \\
$|\bar{\sigma}|$ & $4.69 \times 10^{15}$ & $M_{X}$ & $5.25 \times 10^{17}$ \\
$A_{0}\left(M_{X}\right), m_{0}\left(M_{X}\right)$ & $-5.235 \times 10^{5}, 1.260 \times 10^{4}$ & $\mu, B\left(M_{X}\right)$ & $4.316 \times 10^{5},-1.128 \times 10^{11}$ \\
$M_{\bar{H}}^{2}$ & $-1.498 \times 10^{11}$ & $M_{H}^{2}$ & $-1.448 \times 10^{11}$ \\
$\left|\Delta_{H_{0}}\right|,\left|\Delta_{\bar{H}_{0}}\right|$ & $50.254,63.930$ & $\left|\alpha_{4}\right|$ & 0.707 \\
$M_{3}^{v^{c}}$ & $4.86 \times 10^{13}$ & $M_{1,2}^{v^{c}}$ & $1.181 \times 10^{9}, 2.01 \times 10^{12}$ \\
$\left|y_{31 \text { tree }}^{v}\right|$ & $1.997 \times 10^{-4}$ & $\left|y_{21 \text { tree }}^{v},\right| y_{11 \text { tree }}^{v} \mid$ & $4.489 \times 10^{-5}, 1.640 \times 10^{-6}$ \\
$\log _{10}\left(h^{2} / M\right)$ & -18.706 & $V_{0}, \phi_{\text {end }}$ & $3.579 \times 10^{52}, 2.153 \times 10^{15}$ \\
$N_{\text {pivot }}, N_{C M B}$ & $54.22,4.78 \times 10^{-4}$ & $\Delta, \beta$ & $8.82 \times 10^{-12}, 5.92 \times 10^{-6}$ \\
\hline
\end{tabular}

Due to the large number of heavy fields the dressing of the Higgs fields can be rather large $\left(\sim 10^{2}\right)$. We earlier calculated[14] the dressing for the 10-plet component of the MSSM Higgs. Above we showed that a completely independent line of argument requires that the doublet $H$ be 50\% derived from the 210-plet. Thus the lengthy calculation of the wave function corrections for each of the six GUT doublets contributing to the MSSM doublet is necessary. Even from the partial calculation[14] we see that the large value of the wave function dressing makes the GUT tree level matter fermion yukawa couplings (i.e $\left\{h_{A B}, g_{A B}, f_{A B}\right\}_{\text {tree }}$ and therefore all the $\left.\left(y_{A B}^{f}\right)_{\text {tree }}\right)$ required to match the SIMSSM couplings at $M_{X}^{0}$ much smaller than they would be without these corrections. This has the important consequence of suppressing $d=5 \mathrm{~B}$-violation operators since they depend on these yukawas and have no Higgs line. Since it is the tree level couplings that enter the formulae for the inflaton dynamics in the full GUT it is easier to satisfy eqn.(8). Because of this and the relatively large value of $M \sim M_{3}$ it should be be possible to achieve the required fine tuning using the full wave function dressing[24].

Embedding in the GUT has overturned our naive assumption that the lowest intermediate scale would govern inflation. Instead it is rather the largest. While setting us the problem of finding solutions to the tuning condition, compatible both with an accurate fit of fermion masses and acceptable values of inflationary power spectrum and spectral index, it emphatically shows that the soft terms have little role to play in the fine tuning which belongs rather to the GUT and intermediate scale physics only. Thus the physics of SIMSSM driven inflation is in sharp contrast to the Dirac neutrino mass driven inflation[5, 6] in the MSSM extended by $U(1)_{B-L}$ and right handed neutrinos. Our analysis makes it clear that they lie counterpoised not only as regards the nature of neutrino mass but also as regards the nature of inflation and its regulating mass scale besides their degree of naturalness.

An example of the relevant parameters from an accurate fit of the complete fermion spectrum in the NMSGUT which has also been tuned to make it as compatible as possible with the inflationary scenario presented is seen in Table 1. More details may be found in [28]. The fine tuning between the yukawas proceeds as anticipated with $1-\Gamma=1=\Lambda_{n, d}$. The remaining problem is that $h^{2} / M \sim 10^{-19} \mathrm{GeV}$ is too large. As a result the number of e-folds $N_{C M B}$ is much smaller than required. However as explained the formulae used seriously underestimate the Higgs wave function corrections. Search of the huge parameter space has just begun. We may well hope for a completely realistic fit compatible with inflation in due course. The detailed analysis of reheating behaviour and Lepto-genesis in this model is also underway. Since the fits of fermion masses also yield a value for the CP violation parameter relevant for Lepto-genesis the incorporation of the SSI scenario in the NMSGUT may eventually yield useful additional constraints which will serve to narrow the parameter space further. 


\section{ACKNOWLEDGMENTS}

This is a report of work done in collaboration with Ila Garg and published as [28]. We are grateful to Anupam Mazumdar and Ling Fei Wang for correspondence and collaboration in earlier stages of this work. and thank David Lyth for discussions and useful comments.

\section{REFERENCES}

1. G. F. Smoot et al., Astrophys. J. 396 (1992) L1; E. Komatsu et al. [WMAP Collaboration], Astrophys. J. Suppl. 192 (2011) 18 [arXiv:1001.4538 [astro-ph.CO]].

2. For a recent example, which however requires a modified gravity sector, see e.g I. Masina and A. Notari, Phys. Rev. Lett. 108, 191302 (2012) [arXiv:1112.5430 [hep-ph]]; I. Masina and A. Notari, Phys. Rev. D 85, 123506 (2012) [arXiv:1112.2659 [hep-ph]].

3. For reviews of MSSM flat direction physics see: K. Enqvist and A. Mazumdar, Phys. Rept. 380, 99 (2003); M. Dine and A. Kusenko, Rev. Mod. Phys. 76, 1 (2004). For a recent review of particle physics models of inflation, see: A. Mazumdar, J. Rocher, Phys. Rept. 497, 85-215 (2011). [arXiv:1001.0993 [hep-ph]].

4. A. H. Guth, Phys. Rev. D 23, 347 (1981); A. H. Guth and E. J. Weinberg, Phys. Rev. D 23, 876 (1981).

5. R. Allahverdi, A. Kusenko and A. Mazumdar, JCAP 0707018 (2007) [arXiv:hep-ph/0608138]; R. Allahverdi, B. Dutta and A. Mazumdar, Phys. Rev. Lett. 99261301 (2007) [arXiv:0708.3983 [hep-ph]]

6. S. Hotchkiss, A. Mazumdar and S. Nadathur, JCAP 1106 (2011) 002 [arXiv:1101.6046 [astro-ph.CO]].

7. J. C. Bueno Sanchez, K. Dimopoulos and D. H. Lyth, JCAP 0701 (2007) 015 [hep-ph/0608299]; R. Allahverdi, K. Enqvist, J. Garcia-Bellido, A. Jokinen and A. Mazumdar, JCAP 0706019 (2007) [arXiv:hep-ph/0610134].

8. P. Minkowski, Phys. Lett. B67,110(1977); M. Gell-Mann, P. Ramond and R. Slansky, in Supergravity, eds. P. van Niewenhuizen and D.Z. Freedman (North Holland 1979); T. Yanagida, in Proceedings of Workshop on Unified Theory and Baryon number in the Universe, eds. O. Sawada and A. Sugamoto (KEK,Tsukuba, 1979); R.N. Mohapatra and G. Senjanović, Phys. Rev. Lett. 44, 912 (1980); R.N. Mohapatra and G. Senjanović, Phys. Rev. D23,165 (1981).

9. M. Fukugita and T. Yanagida, Phys. Lett. B 174 (1986) 45.

10. C. S. Aulakh, K. Benakli and G. Senjanovic, Phys. Rev. Lett. 79, 2188 (1997) [arXiv:hep-ph/9703434]; C. S. Aulakh, A. Melfo and G. Senjanovic, Phys. Rev. D 57 (1998) 4174 [arXiv:hep-ph/9707256]; C. S. Aulakh, A. Melfo, A. Rasin and G. Senjanovic, Phys. Lett. B 459, 557 (1999) [arXiv:hep-ph/9902409].

11. C. S. Aulakh, B. Bajc, A. Melfo, A. Rasin and G. Senjanovic, Nucl. Phys. B 597, 89 (2001) [arXiv:hep-ph/0004031].

12. C.S. Aulakh and R.N. Mohapatra, CCNY-HEP-82-4 April 1982, CCNY-HEP-82-4-REV, Jun 1982 , Phys. Rev. D28, 217 (1983); T.E. Clark, T.K.Kuo, and N.Nakagawa, Phys. lett. 115B, 26(1982); C. S. Aulakh, B. Bajc, A. Melfo, G. Senjanovic and F. Vissani, Phys. Lett. B 588, 196 (2004) [arXiv:hep-ph/0306242]; C. S. Aulakh and A. Girdhar, Nucl. Phys. B 711, 275 (2005);B. Bajc, A. Melfo, G. Senjanovic and F. Vissani, Phys. Rev. D 70, 035007 (2004) [arXiv:hep-ph/0402122].

13. C. S. Aulakh and S. K. Garg, The new minimal supersymmetric GUT, arXiv:hep-ph/0612021v1; The new minimal supersymmetric GUT: Spectra, RG analysis and fitting formulae, arXiv:hep-ph/0612021v2; C. S. Aulakh and S. K. Garg, “NMSGUT II: Pinning the Nmsgut@LHC,” arXiv:0807.0917v1 [hep-ph];“NMSGUT II: Fermion Fits and soft Spectra” arXiv:0807.0917v2 [hep-ph]. Merged version of these two papers published as Nucl. Phys.B857 (2012)101, available as arXiv:0807.0917v3.

14. C. S. Aulakh, "NMSGUT-III: Grand Unification upended," arXiv:1107.2963 [hep-ph].

15. A. Arbey, M. Battaglia, A. Djouadi and F. Mahmoudi, arXiv:1207.1348 [hep-ph].

16. D. H. Lyth and E. D. Stewart, Phys. Lett. B 283 (1992) 189.

17. A. R Liddle and S. M Leach, Phys. Rev. D 68 (2003) 103503 [astro-ph/0305263].

18. H. Murayama, H. Suzuki, T. Yanagida and J. Yokoyama, Phys. Rev. Lett. 70 (1993) 1912.

19. J. R. Ellis, M. Raidal and T. Yanagida, Phys. Lett. B 581 (2004) 9 [hep-ph/0303242].

20. G. Felder, L. Kofman and A. Linde, Phys. Rev. D 59 (1999) 123523-1. [arXiv:hep-ph/9812289 ].

21. R. Allahverdi, A. Ferrantelli, J. Garcia-Bellido and A. Mazumdar, Phys. Rev. D 83 (2011) 123507 [arXiv:1103.2123 [hep-ph]].

22. E. J. Ahn and E. W. Kolb, Phys. Rev. D 74, 103503 (2006) [astro-ph/0508399].

23. M. Kawasaki, K.Kohri and T. Moroi , Phys. Rev. D 71,(2005)083502.

24. Charanjit S. Aulakh, Ila Garg and Charanjit Kaur, "Grand Unifcation upended", arXiv: 1107.2693v2(to appear).

25. C. S. Aulakh and S. K. Garg, Nucl. Phys. B 757, 47 (2006) [arXiv:hep-ph/0512224].

26. C.S.Aulakh and A. Girdhar, hep-ph/0204097; v2 August 2003; v4, 9 February, 2004; Int. J. Mod. Phys. A 20, 865 (2005).

27. C. S. Aulakh and A. Girdhar, Nucl. Phys. B 711, 275 (2005) [hep-th/0405074].

28. C. S. Aulakh and I. Garg, Phys. Rev. D 86, 065001 (2012) [arXiv:1201.0519 [hep-ph]].

29. B. D. Wright, arXiv:hep-ph/9404217. 